\title{
TÍNH TOÁN SỰ PHÂN BỐ ỨNG SUẤT XUNG QUANH THÀNH LỖ KHOAN DỰA TRÊN CÁC MÔ HÌNH DÀN HỒI TỔNG QUÁT
}

\author{
HAJAL M., DRAYER M., LÊ PHƯỚC HẢO
}

\section{§1. ĐẶT VẤN Đ商}

Nghiên cứu sự ôn định thành lỗ khoan đặc biệt là các lôx khoan dầu khí là một bài toán ứng dụng mang tính khoa học và kinh tế cao. Sự sập lở thành lỗ khoan sâu nhiều lúc gây hậu quả nghiêm trọng về người và của. Nguyên nhân cừa sự sập lở là do sự cân bằng về lực trong khối đá bị phá hủy, gây nên sự tái phân bố ứng suất khi khoan.

Để giải thích đối xử cơ học của lỗ khoan, người ta sứ dụng nhiều mô hình từ đàn hồi cổ điển đến đàn hồi-déo và dẻo. Tuy nhiên kết quá tính toán theo các mô hình này còn có sự cách biệt quá xa so với các thông số đo đạc tại hiện trường [4]. Có thể nêu hai nguyên nhân chù yếu:

1. Các luật được áp dụng quá đơn giăn để có thể biểu diexn đổi xứ cơ học của một vật liệu phức tạp như đá (các modul đàn hồi, hệ số Poisson dược coi là không đổi, các modul ớ trạng thái chất tăi và dỡ tăi đều bằng nhau, vật liệu được coi là tiêu chuẩn trong miền déo...)

2. Các thí nghiệm nén ba trục quy chuẩn, vần thường được sử dụng, hoàn toàn không thích hợp để mô phỏng đới xử cơ học của đá xung quanh thành lổ khoan. Những hạn chế trên đây thường dẫn đến hai điểm khác biệt lớn giữa tính toán lý thuyết và thực tế:

- Ứng suất tiếp chính lý thuyết $\sigma_{\theta(L x)}$ thường rất lớn so với thực tế (có thể tới 2-3 lần). Nhiều lồ khoan không chống vẫn giữ ổn định lâu dài trong điều kiện $\sigma_{\theta(L T)}$ lớn gấp nhiều lần so với $\sigma_{\theta}$ đo được.

- Giá trị cực đại cúa $\sigma_{\theta(L T)}$ thường nằm ngay trên thành lỗ khoan, trong khi nghiên cứu hình thái học các mánh vỡ cho phép kết luận rằng điểm xuất phát đứt gãy đó có thể năm sâu trong thành lồ khoan.

Kết quả tính toán phân bố ứng suất xung quanh thành lỗ khoan $[1,5]$ và được thực tế xác nhận cho thấy ứng suất chính xuyên tâm $\sigma_{r}$ giảm còn ứng suất chính tiếp tăng so với trạng thái ban đầu (trước khi khoan). Như vậy trạng thái ứng suất xung quanh thành lổ khoan không phài là trạng thái nén ba trục mà là kéo-nén ba trục [2].

Bài báo trình bày kết quá nghiên cứu phân bố ứng suất xung quanh thành lỗ khoan băng phương pháp giải tích trên ba mô hình đàn hồi khác nhau. Ở mối mô hình, kết quả được phân tích và so sánh với mô hình đàn hồi cổ điển và kết quả thực tế hiện trường.

\section{§̧2. BÀI TOÁN}

\subsection{Các phương trình cơ bản}

Giả sư một khối đá nựa vô hạn chịu các ứng suất ngang $\sigma_{n}=P_{c}$ và đứng $\sigma_{d}=\gamma z$, áp lực rống $P_{0}$ và nhiệt độ $T_{o}$ ơ chiều sâu $z$ trước khi khoan. Trạng thái ban đầu này là diểm xuất phát 
để tính biến dạng của đá. Trong khối đá này người ta thi công một lỗ khoan hình trụ thẳng đứng, trục $z$, bán kính $R$, chịu một áp lực $P_{0}$ và nhiệt độ $T_{0}$ ớ chiều sâu $z$.

Xét một lát mớng năm ngang ơ độ sâu $z$ với giả thiết ớ trạng thái ứng suất và biến dạng phăng, Trong hệ tọa độ trụ thì $r, \theta, z$ cũng là các hướng chính của ưng suất và biến dạng.

2.1.1. Úng suất

Đặt $\bar{\sigma}_{r}=\sigma_{r}-P_{c} ; \bar{\sigma}_{\theta}=\sigma_{\theta}-P_{c} ; \bar{\sigma}_{z}=\sigma_{z}-\gamma z$ là các gia số ứng suất so với trạng thái ban đầu $\left(P_{c}, P_{c}, \gamma z\right)$. Ở trạng thái biến dạng phẳng, $\bar{\sigma}_{z}$ là hàm cưa $\bar{\sigma}_{r}, \bar{\sigma}_{\theta}, P, T$, các đại lượng này phụ thuộc duy nhất vào $r$.

Vì vậy các phương trình cân bằng trở thành

$$
\bar{\sigma}_{\theta}=\frac{d}{d r}\left(r \bar{\sigma}_{r}\right)
$$

\subsubsection{Biến dạng}

So với trạng thái ban đầu, các biến dạng trong mặt phẳng $r-\theta$ dược viết:

$$
\varepsilon_{r}=\frac{d u}{d r} ; \quad \varepsilon_{\theta}=\frac{u}{r}
$$

Các phương trình tương thích được viết:

$$
\varepsilon_{\mathrm{r}}=\frac{d}{d r}\left(r \varepsilon_{\theta}\right)
$$

2.1.3. Luật dổi xử cơ học

Luật tổng quát của bài toán là luật đàn hồi - nhiệt - áp tức là được thể hiện trong quan hệ ưng suất - biến dạng - áp lực lỗ rỗng và nhiệt độ:

$$
\varepsilon_{i}=f_{i}\left(\bar{\sigma}_{r}, \bar{\sigma}_{\theta}, \bar{\sigma}_{z}, P, T\right), \quad i=r, \theta, z
$$

Giả thiết về biến dạng phẳng cho phép biểu diễn:

$$
\bar{\sigma}_{z}=f\left(\bar{\sigma}_{\mathrm{r}}, \bar{\sigma}_{\theta}, P, T\right) .
$$

Theo độ thấm, các tính chất nhiệt và các điều kiện biên mà $P$ và $T$ là hàm của $r$, thời gian $t$ nên quan hệ $(2.3)$ đưa về dạng

$$
\varepsilon_{i}=F_{i}\left(\bar{\sigma}_{r}, \bar{\sigma}_{\theta}, r, t\right), \quad i=r, \theta
$$

\subsection{Lời giải}

Rút $\bar{\sigma}_{\theta}$ từ phương trình (2.1) và thay vào $(2.4)$ :

$$
\varepsilon_{i}=G_{i}\left(\bar{\sigma}_{r}, \frac{d \bar{\sigma}_{r}}{d r}, r, t\right) \quad i=r, \theta
$$

Thay $\varepsilon_{\mathrm{r}}$ và $\varepsilon_{\theta}$ bằng các biểu thức (2.5) vào phương trình tương thích (2.2):

$$
\frac{1}{r}\left(G_{r}-G_{\theta}\right)=\frac{\partial F_{\theta}}{\partial \bar{\sigma}_{r}} \frac{d \bar{\sigma}_{r}}{d r}+\frac{\partial F_{0}}{\partial\left(\frac{\partial \bar{\sigma}_{r}}{\partial r}\right)} \frac{d^{2} \bar{\sigma}_{r}}{d r^{2}}+\frac{\partial F_{\theta}}{\partial r}
$$

là phương trình vi phân bậc hai theo $\bar{\sigma}_{r}$ có dạng

$$
\frac{d^{2} \bar{\sigma}_{r}}{d r^{2}}+H\left(\bar{\sigma}_{r}, \frac{d \bar{\sigma}_{r}}{d r}, r, t\right)=0
$$

Khi $r \rightarrow \infty$ thì $\bar{\sigma}_{r}$ và̀ $\bar{\sigma}_{\theta} \rightarrow 0$ và $u \rightarrow 0$ kéo theo $r \bar{\sigma}_{r} \rightarrow 0$ 
Các điều kiện biển được viết

$$
\begin{aligned}
& r \rightarrow \infty, \quad r \bar{\sigma}_{r} \rightarrow 0 \\
& r \rightarrow R, \quad \bar{\sigma}_{r}=P_{0}-P_{c}
\end{aligned}
$$

Việc giải phương trình vi phân (2.6) là dễ dàng thực hiện nhờ phương pháp số. Tuy nhiên sự hiểu biết các tính chất của đá đ̛ơ độ sâu vẫn còn rất hận chế, vì vậy trong các luật đối xừ cơ học chỉ nên đưa vào các thông số chử yếu, ảnh hương quyết định đến sự ổn định thành lồ khoan sâu. Sau đây sẽ xét lần lượt một số các yếu tố đó trong lời giải giải tích cưa bài toán.

a. ớ chế độ chất tái và dỡ tái, các modul đàn hồ của đá là không đồi nhưng khác nhau

Như đã trình bày ơ phần trên, trong quá trình khoan, $\sigma_{r}$ giảm (dã tăi) và $\sigma_{o}$ tăng (chất taii) so với trạng thái ban đầu. Thực nghiệm chỉ ra răng các mô đuni ở chế độ chất tăi $\left(E_{c}\right)$ và dỡ tải $\left(E_{d}\right)$ là khác nhau. Tý số $\frac{E_{d}}{E_{c}}$ có thể thay đởi trong phạm vi lớn (tù̀ 1 đến 5 là̀n [5]). Các hệ số Poisson $\left(\nu_{c}, \nu_{d}\right)$ cũng khác biệt nhưng ở mức độ bé hơn.

Trong trường hợp biến dạng phẳng và với $P=0$ và $T=0$, luật đới xứ cơ học được viết:

$$
\begin{aligned}
& \varepsilon_{r}=\frac{1-\nu_{c} \nu_{d}}{E_{d}} \bar{\sigma}_{r}-\frac{\nu_{c}\left(1+\nu_{c}\right)}{E_{c}} \bar{\sigma}_{\theta} \\
& \varepsilon_{\theta}=\frac{-\nu_{d}\left(1+\nu_{c}\right)}{E_{d}} \bar{\sigma}_{r}+\frac{1-\nu_{c}^{2}}{E_{c}} \bar{\sigma}_{\theta}
\end{aligned}
$$

Phương trình (2.6) trở thành

$$
\frac{r^{2} d^{2}\left(r \bar{\sigma}_{r}\right)}{d r^{2}}+\frac{1}{1-\nu_{c}}\left[\left(1-\frac{E_{c}}{E_{d}} \nu_{d}\right) r \frac{d\left(r \bar{\sigma}_{r}\right)}{d r}-\frac{E_{c}}{E_{d}} \frac{1-\nu_{c} \nu_{d}}{E_{c}} r \bar{\sigma}_{r}\right]=0
$$

Đây là phương trình tuyển tính thuần nhất (Euler). Với điều kiện biên $(2.7)$, nghiệm có thể được viết:

$$
\begin{aligned}
& \bar{\sigma}_{r}=-\left(P_{c}-P_{0}\right)\left(\frac{R}{r}\right)^{1+m} \\
& \bar{\sigma}_{\theta}=-m \bar{\sigma}_{r}
\end{aligned}
$$

với

$$
\begin{gathered}
m=B+\left(B^{2}+C^{2}\right)^{1 / 2} \\
B=\frac{\nu_{c} E_{d}-\nu_{d} E_{c}}{2 E_{d}\left(1-\nu_{c}\right)}, \quad C=\frac{E_{c}}{E_{d}} \cdot \frac{1-\nu_{c} \nu_{d}}{1-\nu_{c}^{2}}
\end{gathered}
$$

Khi $1 \leq \frac{E_{d}}{E_{c}} \leq 5$ và $1 \leq \frac{\nu_{d}}{\nu_{c}} \leq 1,5$ thì $\frac{1}{2}<m \leq 1$. Trường hợp $m=1$ tương ứng với lời giải của lý thuyết đàn hồi tuyến tính $\left(E_{c}=E_{d}, \nu_{c}=\nu_{d}\right)$

So với lời giải cổ điển' một số nhận xét sau đây được đưa ra:

- Gia số ứng suất nhó,

- Trị số ứng suất tiếp chính $\sigma_{\theta}$ tại thành lôx khoan giăm đáng kẻ.

- Yếu tố ảnh hường chính đến sự tái phân bố ứng suất là tý số $\frac{E_{d}}{E_{c}}$, còn $\frac{\nu_{d}}{\nu_{c}}$ chỉ đóng vai trò rất nhớ, có thể bó qua được

b. Tái bền tuyến tính với các modul khác nhau ở chế độ chất tải và dơ tải

Ngoài các giả thiết đã nêu ở phần trên, còn có thêm những giả thiết sau đây:

- Vật liệu tái bền tuyến tính, khi đạt tới một ngưỡng dạng Mohr - Coulomb và được dặc trưng bằng $C_{0}$ và $\phi_{0}$.

- Quá ngưỡng tái bền, vật liệu vần giữ tuyến tính nhưng với các modul tái bền khác nhau ở chế độ chất tải và dỡ tăi $\bar{E}_{c}, \bar{\nu}_{c}, \bar{E}_{d}, \bar{\nu}_{d}($ hinh 1$)$ 


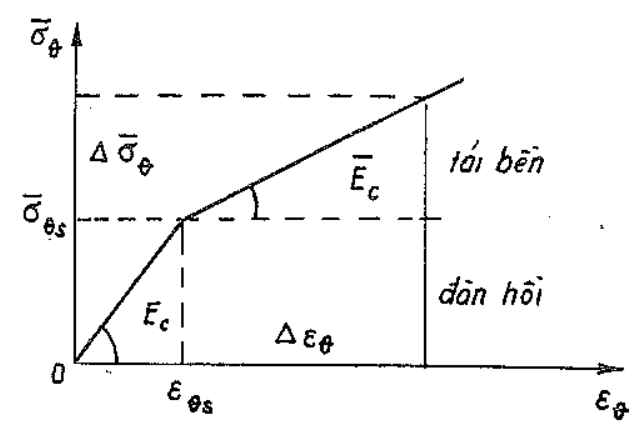

Hinh 1. Tái bền tuyến tính

$$
\begin{aligned}
& \sigma_{\theta}-\sigma_{r}=\sin \phi_{o}\left(\sigma_{\theta}+\sigma_{r}+2 H_{0}\right) \\
& H_{0}=C_{0} \operatorname{cotg} \phi_{o}
\end{aligned}
$$

Ký hiệu với chl số $s$ chl các giá trị cưa các đại lượng ở ngưỡng tái bền. Để tính $\bar{\sigma}_{r s}$ và $\bar{\sigma}_{\theta s}$ tại một điểm ̛ơ khoảng cách $r$, ta xét điểm đó ớ giới hạn vùng đàn hồi:

$$
\bar{\sigma}_{\theta s}=-n \bar{\sigma}_{r s} \quad \text { (phương trình }(2.9) \text { ) }
$$

và ở ngưỡng tái bền

$$
\bar{\sigma}_{\theta s}-\vec{\sigma}_{r s}=\sin \phi_{o}\left(\bar{\sigma}_{\theta s}+\bar{\sigma}_{r s}+2 P_{0}+2 H_{0}\right) \quad \text { (phurơng trình (2.10)) }
$$

Cuối cùng ta rút ra

$$
\begin{aligned}
& \bar{\sigma}_{r s}=-2 \sin \phi_{o} \frac{P_{0}+H_{0}}{\left(1+\sin \phi_{o}\right) m\left(1-\sin \phi_{o}\right)} \\
& \bar{\sigma}_{\theta s}=-m \bar{\sigma}_{r s} \\
& \varepsilon_{r s}-\varepsilon_{\theta s}=\bar{\sigma}_{r s}\left(\frac{1+\nu_{d}}{E_{d}}+m \frac{1+\nu_{c}}{E_{c}}\right)
\end{aligned}
$$

Krý hiệa $\Delta$ là gia số tính từ ngưỡng tái bền, tức là:

$$
\varepsilon_{i}=\varepsilon_{i s}+\Delta \varepsilon_{i ;} \quad \bar{\sigma}_{i s}+\Delta \bar{\sigma}_{i} ; \quad i=r, \theta .
$$

Trong phần tái bền ta có:

$$
\begin{aligned}
& \Delta \varepsilon_{r}=\frac{\left(1-\bar{\nu}_{c} \nu_{d}\right)}{\bar{E}_{d}} \Delta \bar{\sigma}_{r}-\frac{\bar{\nu}_{c}\left(1+\nu_{c}\right)}{\bar{E}_{c}} \Delta \bar{\sigma}_{\theta} \\
& \Delta \varepsilon_{\theta}=\frac{-\nu_{d}\left(1+\bar{\nu}_{c}\right)}{\bar{E}_{d}} \Delta \bar{\sigma}_{r}+\frac{\left(1-\bar{\nu}_{c}^{2}\right)}{\bar{E}_{c}} \Delta \bar{\sigma}_{\theta} .
\end{aligned}
$$

Các phương trình cân băng và tương thích:

$$
\begin{aligned}
& \Delta \bar{\sigma}_{\theta}=\frac{d}{d r}\left(r \Delta \bar{\sigma}_{r}\right)+\left(\bar{\sigma}_{r s}-\bar{\sigma}_{\theta s}\right) \\
& \frac{d}{d r}\left(r \Delta \varepsilon_{\theta}\right)=\Delta \varepsilon_{r}+\left(\varepsilon_{r s}-\varepsilon_{\theta s}\right)
\end{aligned}
$$

Thay các biểu thức (2.11), (2.12) và (2.13) vào (2.14) ta nhận được phương trình vi phân theo $\Delta \bar{\sigma}_{r}$ 


$$
\begin{aligned}
& \left(1-\bar{\nu}_{c}\right) r^{2} \frac{d}{d r^{2}}\left(r \Delta \bar{\sigma}_{r}\right)+\left(1-\bar{\nu}_{c} \frac{\bar{E}_{c}}{\bar{E}_{d}}\right) r \frac{d}{d r}\left(r \Delta \bar{\sigma}_{r}\right)-\frac{\bar{E}_{c}}{\bar{E}_{d}} \frac{1-\bar{\nu}_{c} \bar{\nu}_{d}}{1+\bar{\nu}_{c}}\left(r \Delta \bar{\sigma}_{r}\right) \\
& =r \bar{\sigma}_{r s}\left[\left(\frac{\bar{E}_{c}}{E_{d}} \frac{1+\nu_{d}}{1+\bar{\nu}_{c}}-1\right)+m\left(\frac{\bar{E}_{c}}{E_{c}} \frac{1+\nu_{c}}{1+\bar{\nu}_{c}}-1\right)\right]
\end{aligned}
$$

Đây là phương trình tuyến tính có vế phải. Nghiệm tổng quát cưa nó là tổng của nghiệm tổng quát của phương trình không có vế phải dạng $\left(a r^{-p}+b r^{-q}\right)$ và một nghiệm đặc biệt của phương trình có vế phải ( $\Delta \sigma_{r}=$ const)

Các điều kiện biên là:

Khi $\quad r=\dot{R}$ thì $\bar{\sigma}_{r}=P_{z}-P_{c}$

$r=r_{1}$ (giới hạn giữa miền đàn hồi và miền tái bền) thì $\bar{\sigma}_{r}$ và $\bar{\sigma}_{\theta}$ là liên tục (do vậy $\varepsilon_{r}, \varepsilon_{\theta}$ và $u$ cũng liên tục).

Tích phân phương trình (2.15) và chú ý đến các điều kiện biên ta nhận đưược:

Trong miền đàn hồi với $r \geq r_{1}$ :

$$
\begin{aligned}
& \sigma_{r}=P_{c}+\bar{\sigma}_{r s}\left(\frac{r_{1}}{r}\right)^{1+m} \\
& \sigma_{\theta}=P_{c}-m \bar{\sigma}_{r s}\left(\frac{r_{1}}{r}\right)^{1+m}
\end{aligned}
$$

Trong miền tái bền với $R \leq r \leq r_{1}$ :

$$
\begin{aligned}
& \sigma_{r}=P_{c}+\bar{\sigma}_{r s}\left[D-a\left(\frac{r_{1}}{r}\right)^{1+m_{1}}-b\left(\frac{r_{1}}{r}\right)^{1+m_{2}}\right] \\
& \sigma_{\theta}=P_{c}+\bar{\sigma}_{r s}\left[D+m_{1} a\left(\frac{r_{1}}{r}\right)^{1+m_{1}}+m_{2} b\left(\frac{r_{1}}{r}\right)^{1+m_{2}}\right]
\end{aligned}
$$

với

$$
\begin{aligned}
& \bar{B}=\frac{\bar{\nu}_{c} \bar{E}_{d}-\bar{\nu}_{d} \bar{E}_{c}}{2 \bar{E}_{d}\left(1-\bar{\nu}_{c}\right)} ; \quad \bar{C}=\frac{\bar{E}_{c}}{\bar{E}_{d}} \frac{1-\bar{\nu}_{c} \bar{\nu}_{d}}{1-\bar{\nu}_{c}^{2}} \\
& m_{1}=\bar{B}+\left(\bar{B}^{-2}+\bar{C}\right)^{1 / 2} ; \quad m_{2}=\bar{B}-\left(\bar{B}^{-2}+\bar{C}\right)^{1 / 2} \\
& D=1+\frac{\left(1+\bar{\nu}_{c}\right)\left[\left(\frac{E_{c}}{E_{d}} \frac{1+\nu_{d}}{1+\bar{\nu}_{c}}-1\right)+m\left(\frac{\bar{E}_{c}}{\bar{E}_{c}} \frac{1+\nu_{c}}{1+\bar{\nu}_{c}}-1\right)\right]}{1-\frac{\bar{E}_{c}}{\bar{E}_{d}}+\bar{\nu}_{c}\left(1-\frac{\bar{\nu}_{c}}{\bar{\nu}_{d}} \frac{\bar{E}_{c}}{\bar{E}_{d}}\right)} \\
& a=-\frac{\left(m-m_{2}\right)+D\left(1+m_{2}\right)}{\left(m_{1}-m_{2}\right)} ; \quad b=\frac{\left(m-m_{1}\right)+D\left(1+m_{1}\right)}{\left(m_{1}-m_{2}\right)}
\end{aligned}
$$

Bán kính giới hạn giữa miền đàn hồi và miền tái bền được xác định:

$$
a\left(\frac{r_{1}}{R}\right)^{1+m_{1}}+b\left(\frac{r_{1}}{R}\right)^{1+m_{3}}=D\left(\frac{P_{c}-P_{0}}{\bar{\sigma}_{r s}}\right)
$$

Xử lý bằng số kết quả trên đây cho thấy:

- Ánh hưởng của hệ số Poisson là bé nhưng sự annh hưởng cửa các tý số các modul và ngưỡng tái bền đến trị số ứng suất là đáng kể.

- So với mô hình đàn hồi tuyến tính:

- Ứng suất tiếp chính giảm đáng kể;

- Để đảm bảo sự ổn định (giới hạn) thành lỗ khoan thì tỷ lệ giữa các áp lực $P_{0}$ (áp lực cột dung dịch khoan) trong hai trường hợp giảm từ 1 đến 1,4 lần. 
c. Modul đàn hồi phư thuộc vào áp lực thành (đàn hö̀ phi tuyến)

Xuất phát từ những gợi ý thực nghiệm và kết quả đo được tại hiện trường, Santarelli và Brown [5] đã đề nghị tính toán phân bố ứng suất xung quanh thành lồ khoan theo mô hình đàn hồi có modul Young phụ thuộc vào áp lực thành $\sigma_{r}$ (hệ số Poisson coi như không đổi).

Với điều kiện trên, quạn hệ giữa các ứng suất chính sẽ được biểu diễn bằng

$$
\sigma_{\theta}=\frac{\nu}{1-\nu} \sigma_{r}-\frac{E\left(\sigma_{r}\right)}{1-\nu} \int \frac{d \sigma_{r}}{E\left(\sigma_{r}\right)}
$$

Nếu như modul đàn hồi được biểu diễn theo quy luật cứa Kulhawy [3]:

$$
E\left(\sigma_{r}\right)=E_{0} \sigma_{r}^{\alpha}
$$

với $E_{0}$ là modul đàn hồi được xác định ̛̛ thí nghiệm nén đơn trục

$\alpha$ - hệ số đặc trung cho tính phi tuyến của vật liệu

thì các ứng suất chính xuyên tâm và tiếp tuyến chaẩn hóa $\left(\sigma_{r n}=\frac{\sigma_{r}}{\sigma_{c}} ; \sigma_{\theta n}=\frac{\sigma_{\theta}}{\sigma_{c}}\right.$ vơi $\sigma_{c}$ là sức kháng nén đơn trục của vật liệu) dược tính theo các biểu thức sau:

$$
\begin{aligned}
& \left.\sigma_{r n}=\left\{\left[\left(\frac{P_{0}}{P_{c}}\right)^{1-\alpha}-1\right]\left(\frac{r}{R}\right)^{(1-\alpha) k}+1\right]\right\}^{\frac{1}{1-\alpha}} \\
& \sigma_{\theta n}=(k+1) \sigma_{r n}-k \sigma_{r n}^{\alpha}
\end{aligned}
$$

với $\quad k=\frac{(1-\alpha)(2 \nu-1)-1}{(1-\nu)(1-\alpha)}$

Dễ nhận thấy rằng biểu thức của Kulhawy có hai nhược điểm sau:

- Khi áp lực thành bé $\left(\sigma_{r} \rightarrow 0\right)$ thì modul đàn hồi cửa vật liệu rất bé $(E \rightarrow 0)$, điều này không đúng với thực tế;

- Biểu thức không cùng thứ nguyên. Để khắc phục nhược điểm trên, biểu thức sau đây đã được đề nghị [1]:

$$
E\left(\sigma_{r}\right)=E_{0}\left(1+\frac{\sigma_{r}}{\sigma_{c}}\right)^{\alpha}
$$

Tuy nhiên, lời giải đúng không phải lúc nào cũng nhận được vì biểu thức $E\left(\sigma_{r}\right)$ nằm dưới dấu tích phân. Chẳng hạn với biểu thức (2.22) nghiệm giải tích chỉ nhận được với trường hợp vật liệu không nén được $(\nu=0,5)$ và khi $\alpha=0,5[1]$. Bài toán này được giăi dễ dàng bằng phương pháp số với quan hệ phụ thuộc $E\left(\sigma_{r}\right)$ được xác định băng các thí nghiệm kéo - nén ba trục đồng thời $[2]$.

Kết quả tính toán bằng số theo mô hình này cho thấy:

- Ứng suất chính tiếp $\sigma_{\theta}$ giảm đáng kể và giá trị cực đại của nó không nhất thiết nằm ngay trên thành lỗ khoan như trong trường hợp vật liệu đàn hồi tuyến tính mà có thể nằm sâu trong thành lỗ khoan. Đây là ưu điểm nởi bật của mô hình này;

- Trị số ứng suất và biến dạng phụ thuộc rất lớn vào hệ số $\alpha$ đặc trưng độ phi tuyến của vật liệu

\section{§3. KẾT LUẬN}

Việc nghiên cứu sơ bộ bài toán phân bố ứng suất xung quanh thành lồ khoan đã chỉ ra rằng:

1. Dù luật đối xử cơ học vật liệu có phức tạp vẫn có thể giải được bài toán phẳng. Mọi giả thuyết không hiện thực để đơn giản hóa bài toán sẽ không kiểm chứng được.

2. Trong luật đới xử cơ học nhất thiết phải tính đến:

- Sự phụ thuộc của modul đàn hồi vào áp lực thành. 
- Sự thay đổi modul khi có tái bền.

- Các modul khác nhau ờ chế độ dỡ tải và chất tải.

Các thông số này ảnh hưởng đáng kể đến trị số các ứng suất. Đối với các hệ số Poisson, sự ảnh hường này có thể bó qua được.

3. Thí nghiệm nén' ba trục quy chuẩn không thích hợp với bài toán lỗ khoan, do vậy cần thay băng dạng thí nghiệm kéo - nén ba trục đồng thời với việc mô phơng điều kiện đáy lỗ khoan.

Các mô hình đàn hồi có tính đến ảnh hướng của đ̛̣ường chất tải lên tînh chất cơ hợc của đá được trình bày trên đây đã cho kết quả tương đối phù hợp với thực tế: cụ thể là trị số ứng suất chính tiếp $\sigma_{\theta}$ trên thành lỗ khoan giảm đáng kể và giá trị cực đại của nó không nhất thiết nằm ngay trên thành như lời giải cổ điển mà có thể nằm sâu trong thành lỗ khoan. Các lời giải này là cơ sở để so sánh và đối chiếu với lời giải bài toán tổng quát bằng phương pháp số.

Dịa chi:

Nhận $n g \dot{a} y$ 8/5/1993

Trương DHTH Beyrouth (Liban)

Trứng DHTH Joseph Fourier (France)

Trường $D H B K$ Tp. HCM

\section{TÀI LIỆU THAM KHẢO}

1. Le Phuoc H. Distribution des contraintes autour des forages profonds. Mémoire de DEA, Université Joseph Fourier, Grenoble. 1988.

2. Le Phuoc H. Comportement des roches autour d'un forage: cas particulier de deux calcaires poreux. Thèse de Doctorat de l'Université Joseph Fourier, Grenoble. 1991.

3. Kulhawy F. W. Stress Deformation of Rock and Rock Discontinuities Engng. Geol. No 9, $1975,327-350$.

4. Maury V. Observation, recherche et résultat récents sur les mécanismes de rupture autour de galéries isolées. Rapport de la commission SMIR sur les mécanismes de rupture autour d'ouvrages souterrains. $6^{\text {eme }}$ congrès Int. de Mécanique des roches, Montréal, 1987.

5. Santarelli E. I., Brown E. T. Performance of Deep Wellbores in Rock with a Confining Pressure - Dependent Elastic Modulus. Proc. $6^{\text {th }}$ Congr. Int. Soc. Rock Mech., Montreal, vol. 2, $1987,1217.1222$.

\section{RESUME}

\section{CALCUL ANALYTIQUE DE DISTRIBUTION DES CONTRAINTES AUTOUR DE FORAGE BASÉ SUR LES MODĖLES ÉLASTIQUES GÉNÉRALISÉS}

L'árticle résume les principales causes donnant l'écart important, dans un problème de forage, entre les modèles élastique linéaire classique ou plastique et les observations in-situ.

Trois solutions analytiques complètes pour les contraintes sont présentées dans le cas d'un puits axisymétrique et dans les roches éclastiques dont les modules sont en fonction du chemin de chargement ou de la pression de confinement. Ces théories qui sont une généralisation de l'élasticité linéaire classique permettent d'expliquer convenablement la résistance "anormale" energistrée et le modes de rupture à la poroi de puits profond. 